\title{
Statistical inferences for bearings life using sudden death test
}

\author{
Cristin-Olimpiu Morariu ${ }^{1, *}$, and Sebastian-Marian ZAHARIA ${ }^{1}$ \\ ${ }^{1}$ Transilvania University of Brasov, Department of Manufacturing Engineering, 29 Eroilor Street, \\ Brașov, Romania
}

\begin{abstract}
In this paper we propose a calculus method for reliability indicators estimation and a complete statistical inferences for three parameters Weibull distribution of bearings life. Using experimental values regarding the durability of bearings tested on stands by the sudden death tests involves a series of particularities of the estimation using maximum likelihood method and statistical inference accomplishment. The paper detailing these features and also provides an example calculation.
\end{abstract}

\section{Introduction}

The durability / reliability tests of the bearings are made with specialized tests machines in order to meet the following conditions:

- ensuring identical operating conditions for all bearings tried;

- to reproduce the operating conditions of the bearings in accordance with the assumptions for calculating the basic radial load (moderate speeds, charging with a pure radial/axial load for ensuring a proper lubrication by using recommended lubricants, that are uncontaminated or chemically and mechanically degraded, to provide cooling, the inner ring rotating and outer ring fixed) and to permit the easy mounting and dismounting of bearings;

- to be provided with equipment for measuring temperature and vibration, in order to detect the moment when the damage begins;

Also, when manufacturing the bearings, their testing is achieved by trying to control the indicators of reliability. This test is called type test and it is done at well-defined intervals of time. This type of test verifies if the nominal bearing durability is maintained at the levels required by regulations. Their role is to conduct the audit of production quality through the global assessment of the quality of materials used and technological methods applied.

The main indicator of reliability of bearings, besides the Weibull distribution parameters, is the basic rating life, $L_{10}$, for a bearing or for a group of identical bearings which operate under the same conditions and durability associated with a reliability of $90 \%$, at a conventional material quality manufacturing and operating conditions:

\footnotetext{
* Corresponding author: c.morariu@unitbv.ro
} 


$$
L_{10}=\gamma+\eta \cdot\left[\ln \left(\frac{1}{1-0.10}\right)\right]^{1 / \beta}
$$

In other words, nominal durability is the $10^{\text {th }}$ quantile of the functioning period, $t_{0,10}$, depending on the test procedure used in the manufacturing process of bearings, there can be used:

- Censored tests. An attempt is considered censored at the level $r$, if it ends at the moment $t_{r}$, when the $\mathrm{r}^{\text {th }}$ failure occurs (the number of failures $r$ is established prior to the beginning of attempts).

- Truncated tests. In this case of reliability test, the attempts end after covering a period $t_{c}$, which was previously established.

- Sudden death attempts, $[1,2,3]$. The method consists in random grouping of the $n$ products that are tested, in $\ell$ subsets and taking every subset as an assemble of $m(n=$ $\ell \cdot m$ ) series elements. (Figure 1.).

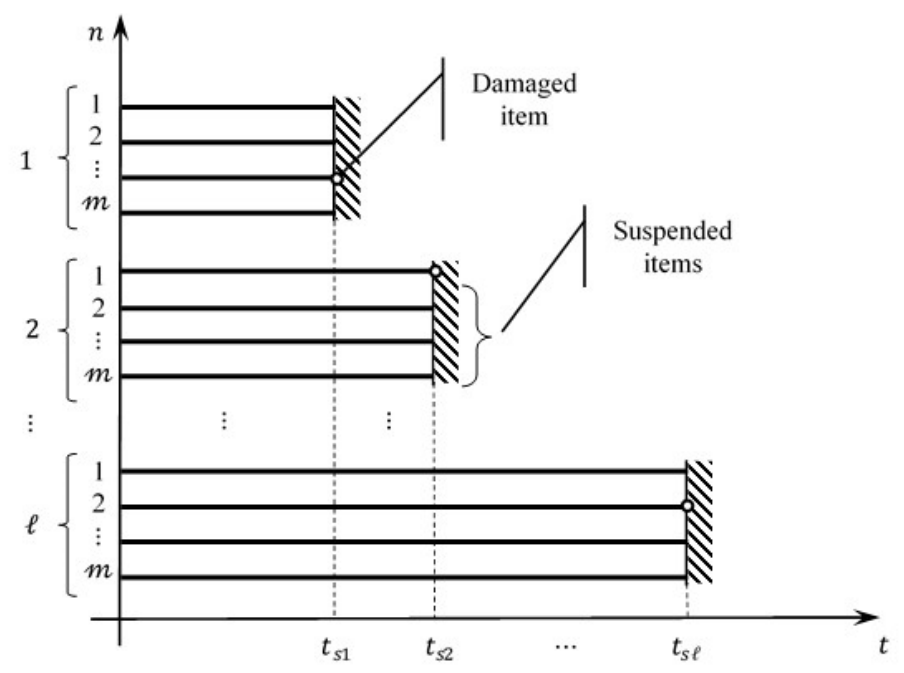

Fig. 1. Deployment scheme of sudden death tests

After making such a grouping, the tests continues until the first appearance and registration of an damaged element from each assemble, the other e $m-1$ elements will be suspended.

The registered deterioration times $t_{s 1}, t_{s 2}, \ldots, t_{s \ell}$ represent the first statistic order in the case of an sample of volume $m$, taken from an Weibull population. If the $n$ elements reveal a Weibull population, then the deterioration times that correspond to the sudden death tests have the distribution function [3]:

$$
F_{S}(t)=1-[1-F(t)]^{m}=1-e^{-\left(\frac{t}{m^{-\frac{1}{\beta} \cdot \eta}}\right)^{\beta}}
$$

If the random variable $T$ has the distribution $\mathcal{W}(t, \beta, \eta)$, then the first statistics of order $T_{s}$ corresponding to an sample of volume $m$ has as distribution $\mathcal{W}\left(t_{s}, \beta, m^{-\frac{1}{\beta}} \cdot \eta\right)$. 
Between the population parameters and the parameters of the first order statistic distribution exist the following relationships [3]:

$$
\left\{\begin{array}{l}
\beta_{s}=\beta \\
\eta_{s}=m^{-\frac{1}{\beta}} \cdot \eta \\
t_{s p}=m^{-\frac{1}{\beta}} \cdot t_{p}
\end{array}\right.
$$

In [3] it is shown that in the case of the maximum likelihood estimators, applied for deterioration times obtained by primary fault line method, there can be established the following random variables, independent of the population parameters:

$$
\left\{\begin{array}{l}
\frac{\hat{\beta}_{s}}{\beta}=v(\ell, \ell) \\
\hat{\beta}_{s} \cdot \ln \left(\frac{\hat{\eta}}{\eta}\right)=s(\ell, m) \\
\hat{\beta}_{s} \cdot \ln \left(\frac{\hat{t}_{0.10}}{t_{0.10}}\right)=q(\ell, m, 0.10)
\end{array}\right.
$$

This procedure is an alternative method used to test the reliability of the bearings $[1,2,3,4]$ which ensures an even distribution of the lower estimated values of reliability indicators. This can be demonstrated by the ratio between the primary line faults dispersion and the dispersion of population [1]:

$$
\frac{V\left(T_{s}\right)}{V(T)}=m^{-\frac{1}{\beta}}
$$

According to the working regime in the case of the bearings reliability tests are used the accelerated attempts. For a shorter attempts period, the constraints are increased, taking into account the operating conditions $[1,2,5]$. The acceleration factor is determined by the formula:

$$
L_{10 h}=\frac{1000000}{60 \cdot n} \cdot\left(\frac{C}{P}\right)^{q}
$$

where:

$n=$ speed test, $[\mathrm{rpm}]$.

According to the volume of products, the specific attempts for bearing manufacturing are attempts done on low volumes of samples.

\section{Special features of estimating the statistical model}

Estimating the Weibull distribution parameters of the statistical model and making inferences for the main statistical indicators of reliability the bearings presents a number of features for tests sudden death.

For an accurate estimation is mainly used the triparametric Weibull distribution.

The location parameter, $\gamma$, is estimated using the correlation coefficient with the condition of using the maximum values possible [5]:

$$
\rho(Y, C)=\frac{\sum_{i=1}^{\ell} \ln \left(t_{s i}-\gamma\right) \cdot c_{i}-\frac{\sum_{i=1}^{\ell} \ln \left(t_{s i}-\gamma\right) \cdot \sum_{i=1}^{n} c_{i} \cdot}{n}}{\left\{\left[\sum_{i=1}^{\ell} \ln ^{2}\left(t_{s i}-\gamma\right)-\frac{\left[\sum_{i=1}^{\ell} \ln \left(t_{s i}-\gamma\right)\right]^{2}}{n}\right] \cdot\left[\sum_{i=1}^{\ell} c_{i}^{2}-\frac{\left(\sum_{i=1}^{\ell} c_{i}\right)^{2}}{n}\right]\right\}^{1 / 2}}
$$


where the deterioration probability values are calculated using the approximate computing relationship, [6,7]:

$$
c_{i}=\frac{1}{1+\frac{n-i+1}{i} \cdot F_{0.50 ; 2 \cdot(n-i+1) ; 2 \cdot i}}, i=\overline{1, n}
$$

Knowing the values of the location parameter, through a transformation like the following one:

$$
X=T_{s}-\gamma
$$

the obtained values are biparametric Weibull distributed.

The punctual estimation method used for finding out the parameters for the Weibull biparametric distribution is the maximum likelihood method. In this case, the estimated values of the shape and scale parameters of the Weibull biparametric distribution are obtained as a solution of the equation, $[6,8]$ :

$$
\left\{\begin{array}{c}
\frac{1}{\hat{\beta}_{s}}+\frac{1}{n} \cdot \sum_{i=1}^{\ell} \ln x_{i}-\frac{\sum_{i=1}^{\ell} x_{i}^{\widehat{\beta}_{s}} \cdot \ln x_{i}}{\sum_{i=1}^{n} x_{i}^{\widehat{\beta}_{s}}}=0 \\
\hat{\eta}_{s} \widehat{\beta}_{s}=\frac{1}{n} \cdot \sum_{i=1}^{\ell} x_{i}^{\widehat{\beta}_{s}}
\end{array}\right.
$$

Based on these estimated values $\left(\hat{\beta}_{s}\right.$ și $\left.\hat{\eta}_{s}\right)$ and using the equations (3), there are determined the population parameters of the tested bearings, $(\hat{\beta}$ și $\hat{\eta})$. The estimated nominal sustainability, $\hat{L}_{10}$, s computed using the equation (1).

For making statistical inferences for estimated parameters are used the distributions of statistics (4). The determination of these distributions is performed by the numerical simulation Monte Carlo. The algorithm used consists of the following steps:

A. Entry data:

- $n$ - sample size;

- $\quad \ell$ - the number of groups into which the sample is divided;

- $m=\frac{n}{\ell}$ - effective sample size;

- $N_{\text {sim }}$ - number of simulations.

B. Generating $n$ random uniform numbers $\left(n_{a i}, i=\overline{1, n}\right)$ in the interval $[0,1]$.

C. Calculating the damaging times $\left(t_{i}\right)$ by using the inverse distribution function:

$$
t_{i}=\ln \left(\frac{1}{1-n_{a i}}\right)
$$

D. Dividing the $n$ random values calculated at the previous step in $\ell$ groups, each one of them having $m$ damaging times.

E. Computing the $\ell$ deterioration times corresponding to the primary fault line from the condition regarding the minimum $m$ durations, corresponding to each group.

F. Estimating the Weibull distribution parameters by taking into account the $\ell$ values previously determined.

Obtaining the maximum likelihood estimates $\left(\hat{\beta}_{1}\right.$ și $\left.\hat{\eta}_{1}\right)$ by iterative numerical solving the system of equations (9) and using the relationships (3);

G. Calculus of 0.10 quantiles, by using the equation (1).

H. Computing the specific random variables:

$$
\hat{\beta}_{1, s}=v(\ell, \ell) ; \hat{\beta}_{1, s} \cdot \ln \hat{\eta}_{1, s}=s(\ell, m) ; \hat{\beta}_{1, s} \cdot \ln \hat{t}_{1,0.10}=q(\ell, m, 0.10)
$$


I. Repeat the steps $\mathrm{A}, \ldots, \mathbf{H}$ for $N_{\text {sim }}$ times.

J. Determining the random variables quantiles by taking into consideration the $N_{\text {sim }}$ made for each case.

K. Output data:

- ASCII file (*.prn) which contains the quantiles values.

On the basis of the original algorithm was carried out a Mathcad program.

If the random variables (4), independent of population parameters, there are known statistical inferences for the Weibull distribution parameters and nominal durability is achieved using the equations:

1. For the shape parameter:

- bilateral symmetric interval, corresponding to a confidence level of $1-\alpha$, is obtained as the solution of the probability equation:

$$
\operatorname{Pr}\left[v_{\alpha / 2}(\ell, \ell) \leq \frac{\hat{\beta}_{s}}{\beta} \leq v_{1-\alpha / 2}(\ell, \ell)\right]=1-\alpha
$$

and it has the form:

$$
\frac{\hat{\beta}_{s}}{v_{1-\alpha / 2}(\ell, \ell)} \leq \beta \leq \frac{\hat{\beta}_{s}}{v_{\alpha / 2}(\ell, \ell)}
$$

- unbiased average estimate:

$$
\hat{\bar{\beta}}=\frac{\hat{\beta}_{s}}{\bar{v}(\ell, \ell)}
$$

- median unbiased estimation:

$$
\hat{\beta}_{M e}=\frac{\hat{\beta}_{s}}{v_{0.50}(\ell, \ell)}
$$

2. For the scale parameter:

- bilateral symmetric interval, corresponding to a confidence level of $1-\alpha$, is obtained as the solution of the probability equation:

$$
\operatorname{Pr}\left[s_{\alpha / 2}(\ell, m) \leq \hat{\beta}_{s} \cdot \ln \left(\frac{\hat{\eta}}{\eta}\right) \leq s_{1-\alpha / 2}(\ell, m)\right]=1-\alpha
$$

and it has the form:

$$
\hat{\eta} \cdot\left[e^{\frac{-s_{1-\alpha / 2}(\ell, m)}{\widehat{\beta}_{s}}}\right] \leq \eta \leq \hat{\eta} \cdot\left[e^{\frac{-s_{\alpha / 2}(\ell, m)}{\widehat{\beta}_{s}}}\right]
$$

- unbiased average estimate:

$$
\hat{\bar{\eta}}=\hat{\eta} \cdot e^{\frac{-\bar{s}(\ell, m)}{\hat{\beta}_{s}}}
$$

- median unbiased estimation:

$$
\hat{\eta}_{M e}=\hat{\eta} \cdot e^{\frac{-s_{0.50}(\ell, m)}{\hat{\beta}_{S}}}
$$

3. For basic rating life, $L_{10}=t_{0.10}$

- bilateral symmetric interval, corresponding to a confidence level of $1-\alpha$, is obtained as the solution of the probability equation: 


$$
\operatorname{Pr}\left[q_{\alpha / 2}(\ell, m, 0.10) \leq \hat{\beta}_{s} \cdot \ln \left(\frac{\hat{t}_{0.10}}{t_{0.10}}\right) \leq q_{1-\alpha / 2}(\ell, m, 0.10)\right]=1-\alpha
$$

and it has the form:

$$
\hat{t}_{0.10} \cdot\left[e^{\frac{-q_{1-\alpha / 2}(\ell, m, 0.10)}{\widehat{\beta}_{S}}}\right] \leq t_{0.10} \leq \hat{t}_{0.10} \cdot\left[e^{\frac{-q_{\alpha / 2}(\ell, m, 0.10)}{\hat{\beta}_{S}}}\right]
$$

- unbiased average estimate:

$$
\hat{\bar{t}}_{0.10}=\hat{t}_{0.10} \cdot e^{\frac{-\bar{q}(\ell, m, 0.10)}{\widehat{\beta}_{s}}}
$$

- median unbiased estimation:

$$
\hat{t}_{0.10, M e}=\hat{t}_{0.10} \cdot e^{\frac{-q_{0.50}(\ell, m, 0.10)}{\widehat{\beta}_{s}}}
$$

\section{Results and discussions}

After testing on the stands a lot of tapered roller bearing $(n=20)$, type LM1 1949/LM11910, random grouped having $\ell=5$ and $m=4$, requested with axial load $P a=450[\mathrm{daN}]$ at $4000[\mathrm{rpm}]$, based on the experimental results, the estimated value of the positioning parameter is:

$$
\hat{\gamma}=63.759[h]
$$

This value was determined in accordance with a coefficient of variation equal to:

$$
\rho(\hat{\gamma})=0.995
$$

which indicates a very good correlation with the Weibull biparametric model.

To achieve statistical inference, further on the random quantiles for the random variables needed to be computed (4).

The following values were obtained:

$$
\begin{array}{lll}
\bar{v}(5,5)=1.448, & \bar{s}(5,4)=-0.686, & \bar{q}(5,4,0.10)=0.328, \\
v_{0.50}(5,5)=1.011, & s_{0.50}(5,4)=-0.347, & q_{0.50}(5,4,0.10)=0.131, \\
v_{0.975}(5,5)=3.522, & s_{0.975}(5,4)=0.683, & q_{0.975}(5,4,0.10)=3.038, \\
v_{0.025}(5,5)=0.617, & s_{0.025}(5,4)=-4.248, & q_{0.025}(5,4,0.10)=-1.149,
\end{array}
$$

under the conditions of $N_{\text {sim }}=10000$ simulations.

Point estimates and confidence intervals for Weibull distribution parameters estimated based on experimental results are presented in Table 1. The confidence level used is:

$$
1-\alpha=0.95 \text {. }
$$

\section{Conclusions}

The paper presents a way to achieving statistical inference for the sudden death tests, more convenient in terms of the operations of assembly and disassembly of bearings on the stand bearings and the accuracy of the experimental results obtained.

Because testing bearings on the stands is done using samples of small volume, making inferences statistics (building confidence intervals and testing statistical hypothesis) is not recommended to use the asymptotic properties of estimates, obtained by maximum 
likelihood method, because the confidence intervals will result having very large amplitudes.

Table 1. Parametric estimation with $95 \%$ confidence interval - Weibull distribution

\begin{tabular}{|c|c|c|c|c|c|c|}
\hline $\begin{array}{c}\text { Weibull } \\
\text { distribution } \\
\text { parameters }\end{array}$ & \multicolumn{6}{|c|}{ Parametric estimation with 95\% confidence interval - Weibull } \\
distribution \\
\hline \multirow{2}{*}{$\begin{array}{c}\text { Shape } \\
\text { parameter, } \beta\end{array}$} & $\hat{\beta}_{s}$ & $\hat{\beta}$ & $\hat{\bar{\beta}}$ & $\hat{\beta}_{M e}$ & $\hat{\beta}_{L}$ & $\hat{\beta}_{U}$ \\
\cline { 2 - 7 } & 1.817 & 1.817 & 1.255 & 1.463 & 0.516 & 2.942 \\
\hline $\begin{array}{c}\text { Scale } \\
\text { parameter, } \eta\end{array}$ & $\hat{\eta}_{s}$ & $\hat{\eta}$ & $\hat{\bar{\eta}}$ & $\hat{\eta}_{M e}$ & $\hat{\eta}_{L}$ & $\hat{\eta}_{U}$ \\
\cline { 2 - 7 } & 157.909 & 338.568 & 494.026 & 409.967 & 232.502 & 3504.769 \\
\hline $\begin{array}{c}\text { Basic rating } \\
\text { life } t_{0.10}\end{array}$ & $\hat{t}_{0.10, s}$ & $\hat{t}_{0.10}$ & $\hat{\bar{t}}_{0.10}$ & $\hat{t}_{0.10, M e}$ & $\hat{t}_{0.10, L}$ & $\hat{t}_{0.10, U}$ \\
\cline { 2 - 7 } & 109.543 & 161.922 & 135.165 & 150.655 & 30.437 & 304.683 \\
\hline
\end{tabular}

In this case, are recommended the random variables (4). Unfortunately the literature does not contain quantiles of these random variables. Therefore, this paper presented an original algorithm for easily calculating the quantiles of these random variables.

Also, the method described is based on Monte Carlo simulation techniques. For this reason, in order to obtain more precise values we need to increase greatly the number of simulations. The minimum recommended number of simulations is $N_{\text {sim }}=1000$ of simulations. The results presented in this paper were obtained on the basis of 10000 of simulations.

\section{References}

1. W. Changsen, Analysis of Rolling Element Bearings, (Mechanical Engineering Publications LTD, London, 1991)

2. T.A. Harris, Rolling Bearing Analysis, 3-rd. Edition (John Wiley \& Sons, Inc., 1991)

3. J.I. McCool, IEEE Transactions on Reliability, 19, 127 (1974)

4. J.I. McCool, Using the Weibull Distribution. Reliability, Modeling, and Inference (John Wiley \& Sons, Inc., 2012)

5. C.O. Morariu, Optimizing the reliability tests of bearings, doctoral thesis, Transilvania University of Brasov (1999)

6. D.B. Kececioglu, Reliability Engineering Handbook (PTR Prentice-Hall, Inc. Englewood Cliffs, New Jersey, 1991)

7. D.B. Kececioglu, Reliability \& Life Testing Handbook (PTR Prentice-Hall, Inc. Englewood Cliffs, New Jersey, 1993)

8. D.B. Kececioglu, Reliability \& Life Testing Handbook (PTR Prentice-Hall, Inc. Englewood Cliffs, New Jersey, 1994) 